\title{
Pack die Sonne in den Tank: Zur Weiterentwicklung nachhaltiger Energiesysteme
}

\author{
Robert Schlögl \\ Fritz-Haber-Institut der MPG, Department of Inorganic Chemistry \\ Faradayweg 4-6, 14195 Berlin \\ Max-Planck-Institut für Chemische Energiekonversion \\ Stiftstr. 34-36, D-45470 Mülheim an der Ruhr
}

\section{Abstract:}

Der Umbau von Energiesystemen hin zu nachhaltigen Lösungen schreitet seit einigen Jahren voran. Damit kann seine Wirkung im Klimaschutz durch Projektionen abgeschätzt werden. Dies geschieht hier für die Region der Europäischen Union. Das Resultat wird mit den Zielen des Pariser Klimavertrages verglichen. Es zeigt sich, dass zusätzliche qualitativ andersartige Maßnahmen ergriffen werden müssen.

Der Umbau von Energiesystemen um Treibhausgase zu vermeiden schreitet voran. Die techno-ökonomische Reife der physikalischen Wandler (PV, Windräder) von Sonnenenergie in elektrischen Strom treibt den Umbau an. Daraus leitet sich die Vorstellung ab, dass der Wandel der Energiesysteme durch Dekarbonisierung, den Verzicht auf Kohlenstoff als materiellem Energieträger erfolgt. Der Einsatz von PV und Windrädern auf systemrelevanten Skalen zeigt allerdings die Grenzen der Belastung des Stromsystems durch die Volatilität auf. Gedämpft wird diese Wirkung durch stabile Erneuerbare Energie-Ressourcen (RES) wie Biomasse und Wasserkraft. Speicherlösungen ${ }^{[1]}$ mit Batterien sind bereits im Einsatz, sie können aber nicht auf die Dimension eines Stromsystems skaliert werden. Diese Dimension wird durch Chemische Energie Konversion CEC erreichbar[2], welche als zentrales Element die Wandlung von Strom zu Wasserstoff aufweist. Dieser wird zu leicht transportier- und speicherbaren molekularen Folgeprodukten[3] gewandelt. Damit wird das Ziel einer Dekarbonisierung teilweise aufgegeben und ein technischer Kohlenstoffkreislauf tritt in einem stabilen nachhaltigen Energiesystem auf. Dieser besteht aus einem Subsystem für die Sammlung und Aufbereitung von $\mathrm{CO}_{2}$ und einem weiteren System von CEC Prozessen, die das $\mathrm{CO}_{2}$ mit Wasserstoff zu neuen Energieträgern umsetzen. Die Familie von Verfahren ${ }^{[4]}$ wird als Carbon Capture and Use (CCU) bezeichnet. Das Ziel der De-fossilisierung muss allerdings weiter gelten, um den technischen Kohlenstoffkreislauf so klein wie möglich zu halten. Diese Bedingung folgt aus dem erheblichen Verlust von Prozesseffizienz ${ }^{[5]}$ wenn man RES in stoffliche Energieträger wandelt.

Damit CEC und Wärmeerzeugung Energiesysteme wirksam stabilisieren können, bedarf es einer gesamthaften Betrachtung[6] mit einem Energieaustausch zwischen den Sektoren Strom, Mobilität, Wärme und Materialsynthese. Diese Betrachtung eliminiert zudem den Einwand[7] einer möglichen „Doppelzählung“ der Vermeidung von $\mathrm{CO}_{2}$. Bisher wird CCU oftmals im Zusammenhang mit dem Ersatz von fossilen Quellen in der chemischen Industrie für die Herstellung von Chemikalien genannt $[4,8]$. So attraktiv diese Option ist, so begrenzt ist ihr Potential für eine klimarelevante Reduktion der Treibhausgasemission. Dieser Teil von CCU wird strategisch bedeutsam ${ }^{[9]}$ in einer Zeit, 
in der fossile Rohstoffe für die chemische Industrie knapp werden. Eine unverzichtbare Rolle spielen CCU Prozesse in Energiesystemen mit geschlossenen Stoffkreisläufen für die Einbindung industrieller Grundprozesse, die nicht ohne die Verwendung von Kohlenstoff oder die Emission von $\mathrm{CO}_{2}$ auskommen können (Zementherstellung, Stahlindustrie etc). Im hiesigen Kontext wird CCU jedoch als ein Teil des Energiesystems mit den großen Komponenten Strom, Mobilität und Wärme betrachtet.

Eine Alternative zu diesem komplexen Ansatz stellt die Kombination von RES und fossilen Trägern gekoppelt mit einer Speicherung des entstanden $\mathrm{CO}_{2}$ in der Geosphäre ${ }^{[10]}$ durch Carbon Capture and Storage (CCS) dar. Dieses System ist wesentlich einfacher, benötigt weniger Energieaufwand und kann aus heute existierenden Technologien im relevanten Zeitraum und in wirksamer Größe realisiert werden. Ein entscheidender Nachteil dieser auch kostengünstigen Lösung ist, dass es sich dabei um kein nachhaltiges System handelt, da der Kohlenstoffkreislauf nicht geschlossen wird und die langfristigen Konsequenzen für die Geosphäre nicht kontrollierbar sind.

In Abbildung 1 sind die entsprechenden Ansätze gegenüber gestellt. Die Zahlenangaben sind modellhaft zur Illustration der relativen Größenordnungen gewählt. Im heutigen Modell sind die Sektoren getrennt und RES wird in der Stromerzeugung eingesetzt. Für 20 RES Einheiten spart man 20 Einheiten an $\mathrm{CO}_{2}$ Emission. Im CCU Modell B wird sehr viel RES benötigt. Die Sektoren sind gekoppelt. Es werden noch 50 Einheiten $\mathrm{CO}_{2}$ aus stabilisierten anderen Sektoren als dem letztlich emittierenden Mobilitätssektor frei gesetzt. Allein für die dafür nötigen CCU Prozesse werden 200 RES Einheiten benötigt. Dies ist mehr, als RES in allen Sektoren direkt eingesetzt wird (170 Einheiten). Für 470 RES Einheiten spart man $250 \mathrm{CO}_{2}$ Einheiten. Dieses Modell hat alleine das Potenzial durch Rückführung des $\mathrm{CO}_{2}$ mittels Biomasse oder durch "direct air capture" DAC ${ }^{[11]}$ weitgehend bis auf die unvermeidlichen Quellen der Materialherstellung nachhaltig zu werden. Die CCU Option wird dann zyklisch. Allerdings steigt der RES Verbrauch dann nochmals an. Im Modell $\mathrm{C}$ wird CCS zur Vermeidung von $\mathrm{CO}_{2}$ Emission eingesetzt. Der geringe Eigenverbrauch der CCS Technologie wird durch 20 RES Einheiten gedeckt. Dann verbleibt eine Restemission von $50 \mathrm{CO}_{2}$ Einheiten für den Einsatz von nur $190 \mathrm{RES}$ Einheiten, die überwiegend direkt in den Sektoren zum Einsatz kommen. Dieses Modell kann allerdings nicht nachhaltig werden, da es den Kohlenstoffkreislauf nicht schließt. Da die Technologieeinheiten "carbon capture and transport" in den Modellen B und C gleich geartet sind, könnte man Modell C als Brückentechnologie zu Modell B einsetzen, bis ausreichend RES verfügbar sind. Damit würde Zeit gewonnen für den Umbau der Energiesysteme aber es verbleiben die Langzeitrisiken, die mit der Nutzung der Geosphäre verbunden sind.

Die Debatte um CCU vs. CCS wird mit hoher Intensität und Emotionalität (,it is a monstrous thermodynamic crime to reduce $\mathrm{CO}_{2}$ ", Paul Fennell in ${ }^{[12]}$ ) geführt. Ein zentrales Element dieser Debatte ist die Bewertung von Effizienzen in den Prozessketten der Energiewandlung. Dieses Feld leidet unter dem Fehlen einer anerkannten Methode zur Definition von Systemgrenzen und unter dem Fehlen validierter Parameter die als Ersatz mit sehr unterschiedlichen Annahmen geschätzt werden. 

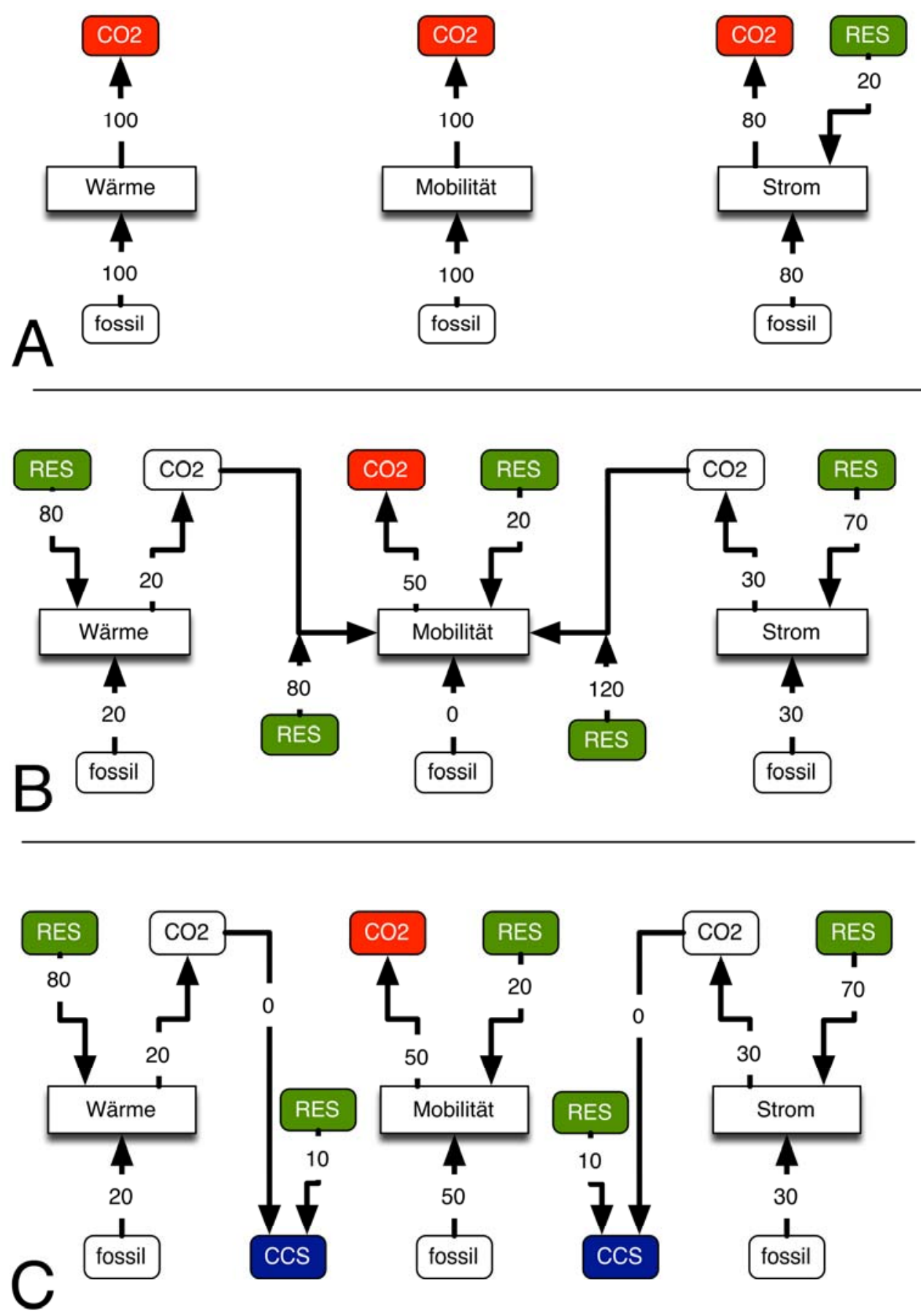

Abbildung 1: Strukturen von Energiesystemen. Die Zahlen sind willkürliche Rechnungseinheiten. Es werden keine Verluste für Prozesse und Transporte berücksichtigt. Effizienzfaktoren sind 2,5 für elektrisches Fahren und 4,0 für CCU. (A) heutige Struktur, (B) ein lineares CCU Modell, (C) ein CCS Modell.

Die Debatte entwickelt sich zu einem Hemmschuh für den Umbau des Energiesystems ${ }^{[5}$, 13], da die zwingend erforderlichen Flexibilisierungsmaßnahmen[6] im Stromsystem von dieser Entscheidung maßgeblich beeinflusst sind. Hier hilft offenbar auch nicht der Vorschlag[14] in beide Richtungen zu gehen um schnellstmöglich mit der gezielten Verringerung der $\mathrm{CO}_{2}$ Emission voranzukommen. 
Die Dringlichkeit, diesen Hemmschuh für die Entwicklung der Energiesysteme zu beseitigen, folgt aus der Betrachtung der bisher erreichten Fortschritte. Für den EU Raum sind aggregierte Kenndaten in Abbildung 2 zusammen gestellt.

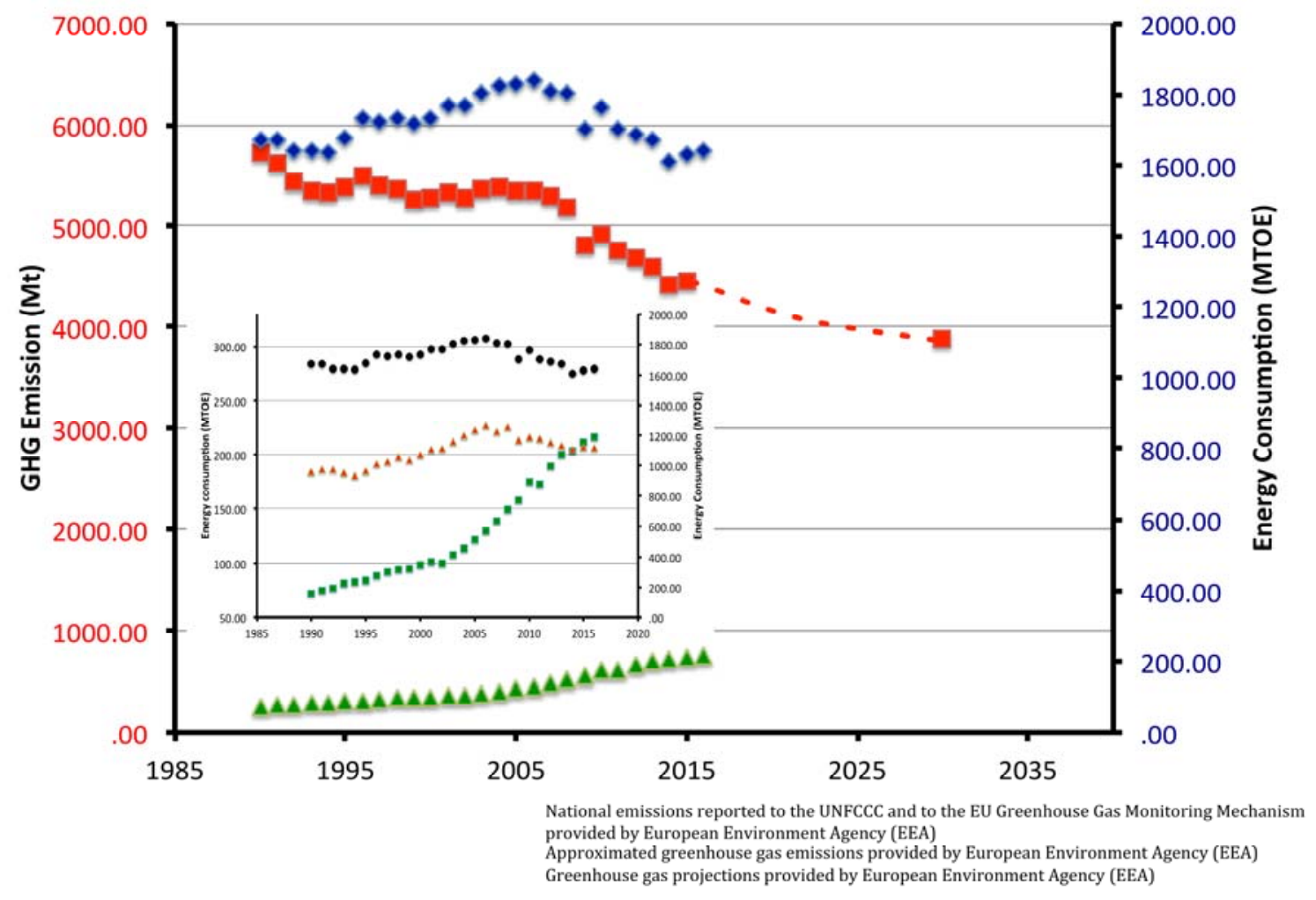

Abbildung 2: Kenndaten der Entwicklung des Energiesystems der EU. Das eingeschobene Bild vergleicht den Gesamtenergieverbrauch (schwarz) mit der Menge an importierten Energieträger (incl. Uran, braun) und den erneuerbaren Energien (grün). Deren zeitlicher Verlauf ist in anderem Maßstab auch in der Hauptabbildung dargestellt.

Die Entwicklung des Energieverbrauches (blau, linke Achse) ergibt, dass heute ein etwa gleich hoher Energiebedarf besteht wie vor 25 Jahren. Diese nicht-lineare Entwicklung überrascht, da sich die Wirtschaft und der Konsum durchaus fortentwickelten. Die Verbesserung der Energienutzung dürfte auf eine Veränderung der Struktur der Wirtschaft im Zuge der Globalisierung zurückzuführen sein. Darauf weist der stetig steigende Energieanteil für Mobilität und Transport hin. Die Treibhausgasemission (rot, rechte Achse) folgte bis vor ca. 10 Jahren der Energiekurve um danach deutlich zurückzugehen. Diese Entwicklung dürfte auf einen Rückgang des Anteil der Produktion an der Wirtschaftsleistung gekoppelt mit einem Rückgang des Kohleanteils im Energiemix, auf die verstärkte Verwendung von Gas als Energieträger, auf die Verbesserung der Energieeffizienz in der Wirtschaft und auf zahlreiche weitere hier nicht zu entschlüsselnde Effekte zurückzuführen sein. Wesentlich hat weiter die verstärkte Nutzung von RES (grün, rechte Achse) beigetragen. Allerdings flacht sich der steile Zuwachs der vergangenen 10 Jahre derzeit langsam ab. Dies zeigt die kleine Grafik zusammen mit der Entwicklung der Importabhängigkeit, die deutlich zugenommen hat. Ursache dafür ist der Wandel des Energiemix weg von Kohle hin zu Öl und Gas. Die EU hat ihre Treibhausgasemission nicht nur durch Änderungen in ihrer Wirtschaftsstruktur verlagert, sondern tatsächlich den Umbau hin zur Nachhaltigkeit soweit vorangetrieben, dass er in der Entwicklung der Treibhausgasemission erkennbar wird. 
Die Europäische Umweltagentur[15] hat diesen Trend auf Grund der Meldungen aller Mitgliedstaaten über ihre Maßnahmen zur Treibhausgasreduktion für die kommenden 15 Jahre prognostiziert und kommt zu dem Schluss, dass sich der Trend zur Reduktion der Treibhausgasemission leicht verstärken wird (siehe Trend in Abbildung 2). Man erwartet einen Rückgang der Treibhausgasemissionen um 32\% bis 2035 bezogen auf das Jahr 1990 und damit eine Verfehlung des Ziels von 40\%. Die Abbildung zeigt weiter, dass diese Fehlentwicklung sich noch wesentlich stärker auf die Erreichung des Klimaziels von $85 \%$ bis 2050 auswirken wird, das massiv verfehlt werden wird. In der Konsequenz zeigt die Abbildung, dass es zusätzlicher drastischer Maßnahmen zur Beschleunigung des Umbaus des Energiesystems bedürfen wird, wobei ein zeitlich linearer Zubau von RES keine ausreichende Maßnahme sein kann.

Es verbleibt die Aufgabe ein nachhaltiges Energiesystem nachvollziehbar zu konzipieren[14, 16]. Ausgehend von den bisher erreichten Veränderungen sollten möglichst viele bestehende Technologien und Infrastrukturen weiter verwendet werden, eine Überlastung von Ressourcen wie Biomasse, Landnutzung und Wasserreserven sollte vermieden werden und Anforderungen an menschliche Verhaltensänderungen wie der Verzicht auf Energienutzung sollten minimalisiert sein. Im Zeitfenster von $1000 \mathrm{Gt} \mathrm{CO}_{2}$ Emissionen muss auf nachhaltige Weise eine ausreichende Reduzierung der Treibhausgasemissionen erreicht werden.

Dieses komplexe Gesamtziel kann nach derzeitigem Wissen neben der Hebung von Effizienzgewinnen in allen Energiesektoren (z.B. Wärmebedarf, Stromerzeugung) nur durch den massiven Einsatz von erneuerbarer Energie in Form von Elektrizität aus Photovoltaik[17] und Wind erreicht werden. Ihr Ausbau wird sich massiv beschleunigen müssen[5]. Damit dies wirksam werden kann, ist das Problem der unpassenden räumlichen und zeitlichen Verteilung der RES im Vergleich zu ihrem Bedarf auf großer Skala zu lösen. Derzeit wird RES vor allem als lokale elektrische Energie gedacht, die über Stromleitungssysteme verteilt wird. In dieser Vorstellung wird elektrische Energie ohne Wandlungsverluste innerhalb des Leitungssystems sofort genutzt. Diese Nutzung lokaler RES ist ein zentrales Element jedes nachhaltigen Energiesystems. Flexibilisierungsmaßnahmen innerhalb der elektrifizierten Nutzung ${ }^{[6]}$ sind möglich und werden umfänglich zu nutzen sein.

Um dies besser als bisher umzusetzen, wird allerdings eine Neubewertung der RES erforderlich. Bisher betrachte man RES als sehr wertvolle Energie, die sparsam und mit maximaler Effizienz einzusetzen ist. Flexibilisierung erzeugt Effizienzverluste und zusätzliche Kosten. Daher vermeidet man diese bisher weitgehend. Die derzeitige Bewertung von RES vernachlässigt das Fehlen von verbrauchsabhängigen Kosten („Brennstoffkosten“). RES verursachen vor allem Infrastrukturkosten für Wandler. Die Verteilkosten sind ähnlich wie bei fossiler Elektrizität. Zur Minimierung der Infrastrukturkosten sind technische Entwicklungen der Wandler und die Entfrachtung von politischen Kosten (Steuern, Subventionen, Auflagen) sehr hilfreich. Zudem ist ihr Betrieb in Gegenden mit hohem Aufkommen von Sonnenergie angezeigt. In Europa[18] ist dies an Küsten und im Süden der Fall, in den Zentren des Verbrauches trifft dies weniger zu und verteuert dort RES.

Somit wird es für den Entwurf stabiler Energiesysteme zur zentralen Herausforderung, RES transportierbar zu machen was auf den erforderlichen Skalen (siehe Abbildung 2) nur durch Umwandlung in chemische Energieträger möglich ist. Wenn dies erreicht 
wird, kann das Subsystem der lokalen RES durch ein Subsystem der transport- und lagerfähigen entfernten RES erweitert werden. Betrachtet man noch einmal Abbildung 2, so sieht man, dass die EU derzeit den Großteil seiner Energie importiert. Unterstellt man, dass in Zukunft die EU ihren regionalen Energieanteil mit lokaler RES produziert und dabei von Effizienzgewinnen profitiert, so sollte die EU ihren Importanteil durch entfernte RES decken. Bildlich wandelt man die Sonnenenergie an Orten wo sie reichlich vorkommt und lokal nicht benötigt wird in stoffliche Energieträger und transportiert sie in Tanks wohin immer sie gebraucht wird. Dies geschieht in Analogie zum heutigen Transport von fossilen Trägern. Diese Idee ist nicht neu und wurde beispielsweise von der DESERTEC Initiative vorgedacht, allerdings mit der unrealistischen Annahme eines Transportes über Stromleitungen.

Die Energieträger werden dabei so gewählt dass ein geschlossener Kohlenstoffkreislauf entsteht oder kohlenstoff-freie Träger benutzt werden. Ausgehen wird man dabei von Wasserstoff [19] dem universellen stofflichen Energiespeicher, der in ausreichender Menge erzeugt werden kann. Die folgende Tabelle 1 gibt einen Überblick über mögliche Optionen.

\begin{tabular}{|c|c|c|c|c|}
\hline & Medium & Träger & Rücktransport & Anmerkungen \\
\hline \multirow{6}{*}{$\mathrm{H}_{2}$} & keines & $\begin{array}{c}\text { Flüssiger } \\
\text { Wasserstoff }\end{array}$ & nein & \\
\hline & $\mathrm{LOHC}$ & $\mathrm{H}_{\mathrm{x}} \mathrm{LOHC}$ & ja & $\begin{array}{l}\text { Mehrere } \\
\text { Derivate }\end{array}$ \\
\hline & $\mathrm{N}_{2}$ & Ammoniak & nein & $\begin{array}{l}\text { Zahlreiche } \\
\text { Derivate }\end{array}$ \\
\hline & $\mathrm{CO}$ & Gas (SNG) & $\mathrm{Ja}$, als $\mathrm{CO}_{2}$ & \\
\hline & $\mathrm{CO}$ & $\begin{array}{c}\text { FT Produkte, } \\
\text { Kohlenwasserstoffe }\end{array}$ & $\mathrm{Ja}$, als $\mathrm{CO}_{2}$ & $\begin{array}{l}\text { Auch höhere } \\
\text { Alkohole }\end{array}$ \\
\hline & $\mathrm{CO}_{2}$ & Methanol & $\mathrm{Ja}$ & \\
\hline
\end{tabular}

Die Tabelle zeigt, dass bei einigen Optionen das Speichermedium zum Ort der Synthese zurücktransportiert werden muss um den Stoffkreislauf zu schließen. Nutzt man Stickstoff als Trägermedium entfällt diese Aufgabe. Bei ihrer Nutzung durch Oxidation ${ }^{[20]}$ muss allerdings große Sorgfalt auf die Vermeidung der Emission von Treibhausgasen wie $\mathrm{NO}_{\mathrm{x}}$ und $\mathrm{N}_{2} \mathrm{O}$ gelegt werden, die ein sehr viel größeres Erwärmungspotenzial als $\mathrm{CO}_{2}$ haben. Eine Diskussion der Vor- und Nachteile dieser Optionen sprengt den Rahmen dieser Arbeit. Der Autor vertritt die Position der Technologieoffenheit und erwartet, dass alle Optionen großtechnisch zum Einsatz kommen können. Die entsprechende Energieforschung sollte daher mit Nachdruck die Entwicklung aller Optionen vorantreiben um zu einer eventuell noch möglichen Technologieführerschaft für neue Energiesysteme in Europa einen Beitrag zu leisten. Derzeit findet diese Entwicklung[21] allerdings rasant in Fernost statt.

Die Ablehnung eines Konzeptes ${ }^{[5,13]}$ stofflich regernativer Energieträger hat die gleichen Wurzeln wie der Streit um den Nutzen von CCU. Sie liegt in der Überbewertung von Prozesseffizienz (z.B. Einsparpotenzial von $\mathrm{CO}_{2}$ ) gegenüber der eigentlich relevanten Systemeffizienz und der unzutreffenden Vorstellung vom besonderen Wert der RES. Das Zielkriterium ist die Nachhaltigkeit des Gesamtsystems. Damit können keine Vergleiche zwischen nicht-nachhaltigen Systemen und deren Effizienzen (Kosten) und einem nachhaltigen System gezogen werden, die in der Ablehnung der CCU Technologie 
eine große Rolle spielen. Es bleibt die Notwendigkeit, die Kostenstruktur so zu gestalten, dass Energie möglichst unbeschränkt allen institutionellen und persönlichen Mitgliedern der Gesellschaft zu angemessenen Preisen zur Verfügung steht. Dieser Aspekt, zu dem technische Entwicklungen, vor allem aber die Gestaltung der Rahmenbedingungen beitragen ${ }^{[22]}$, wird hier nicht weiter verfolgt.

Die Entwicklung der chemischen Technologien und der zugrunde liegenden Katalyse[19] wird bei den Effizienzen der einzelnen Prozesse erhebliche Verbesserungen bringen können. Die Überlegungen zu Abbildung 1 deuten an, dass mit besseren Prozessen und einer flexiblen Kombination von dezentralen und zentralen Subsystemen entscheidende Einsparungen an RES möglich sind. Gleichzeitig ist unser heutiges Wissen ausreichend[9] um mit der skalengerechten Implementierung von ersten Demonstratoren zu beginnen. Voraussetzung dafür ist allerdings die allgemeine Einsicht, dass ein stabiles nachhaltiges Energiesystem eine Kombination aus lokalen und entfernten RES Quellen benötigt. Zweckmäßigerweise wählt man die Struktur der stofflichen (entfernten) RES so aus, dass sie vorhandenen Anwendungen (Verbrennungsmotoren, Turbinen) und Infrastrukturen (Transportsysteme, Verteilsysteme) nutzen. Ein Anreiz zur Tolerierung unvermeidlich höherer Kosten liegt im Zusatznutzen synthetischer Energieträger welche schädliche Nebenwirkungen ihrer Verwendung ausschalten (z.B. Luftverschmutzung durch Abgase). Die radikale Umsteuerung auf eine neue Basistechnologie wie sie derzeit in Japan[21a] vorgenommen wird, ist in Europa kaum ein Modell.

Die Analogie zwischen stofflichen RES aus entfernten Quellen und fossilen Trägern ergibt sich aus Tabelle 2. Zunächst wird klar dass die Anzahl der Prozessschritte zur Entstehung und Nutzung der Energieträger gleich ist. Bemerkenswert ist die Ausnahme von reinem Wasserstoff, dessen vorteilhaft verkürzte Prozesskette allerdings durch die Schwierigkeiten seiner Handhabung mehr als ausgeglichen wird. Tabelle 2 relativiert die Diskussion um die angeblichen Nachteile stofflicher regenerativer Energieträger gegenüber fossilen Trägern durch unvorteilhaft lange Prozessketten wenn man als gemeinsame Bezugsbasis die Sonnenenergie verwendet. Die wesentlichen Schritte von Bildung und Nutzung stofflicher Energieträger sind sehr ähnlich, auch wenn die einzelnen Transformationen chemisch und physikalisch unterschiedlicher Natur sind.

Die farbig unterlegten Felder der Tabelle 2 sind ökonomisch kostenfrei. Die Nutzung der fossilen Energieträger verbilligt sich daher beträchtlich. Die heutige Kostenstruktur von fossilen und regenerativen Trägern bildet diese Gemeinsamkeit nicht ab. Das mag auch daran liegen dass die Vorleistungen der Natur für die fossilen Energieträger bereits in vorgeschichtlicher Zeit erbracht wurden. Die CCU Träger weisen die längste vom Nutzer zu bezahlende Prozesskette auf. Dabei summieren sich jeweils erhebliche Energiebeträge zur Überwindung der kinetischen Barrieren zusätzlich zum thermodynamischen Energiegehalt und erfordern[5] günstig bereit zu stellende RES. Solange RES fälschlicherweise als kostbar eingeschätzt werden, hat die für das Funktionieren des Gesamtsystems entscheidende Option des globalen Transports von entfernter RES keine gute Chance.

\begin{tabular}{|c|c|c|c|c|c|}
\hline $\boldsymbol{N r}$ & Prozess & Fossile Träger & Biomasse & CCU & Wasserstoff \\
\hline $\mathbf{1}$ & Quelle & \multicolumn{3}{|c|}{ Sonnenenergie } & \\
\hline $\mathbf{2}$ & Bildung & $\begin{array}{c}\text { Photosynthese } \\
\text { mit CO2 }\end{array}$ & $\begin{array}{c}\text { Photosynthese } \\
\text { mit CO2 }\end{array}$ & $\begin{array}{c}\text { Wind, PV, } \\
\text { mechanisch }\end{array}$ & $\begin{array}{c}\text { Wind, PV, } \\
\text { mechanisch }\end{array}$ \\
\hline
\end{tabular}




\begin{tabular}{|c|c|c|c|c|c|}
\hline $\mathbf{3}$ & Wandlung I & Fossilisierung & $\begin{array}{c}\text { Ernte zu } \\
\text { Rohbiomasse }\end{array}$ & Wasserspaltung & Wasserspaltung \\
\hline $\mathbf{4}$ & $\begin{array}{c}\text { Wandlung } \\
\text { II }\end{array}$ & $\begin{array}{c}\text { Extraktion, } \\
\text { Raffinerie }\end{array}$ & Bioraffinerie & CEC mit CO2 & ----- \\
\hline $\mathbf{5}$ & Transport & \multicolumn{4}{|c|}{ Schiffe, pipelines, Bahn, LKW } \\
\hline $\mathbf{6}$ & Nutzung & $\begin{array}{c}\text { Strom, Wärme, } \\
\text { Mobilität, } \\
\text { Material }\end{array}$ & $\begin{array}{c}\text { Wärme, } \\
\text { Material }\end{array}$ & $\begin{array}{c}\text { Mobilität, Wärme, } \\
\text { Strom Ergänzung, } \\
\text { Material }\end{array}$ & $\begin{array}{c}\text { Mobilität, Strom } \\
\text { Ergänzung, } \\
\text { Material }\end{array}$ \\
\hline $\mathbf{7}$ & Emission & $\begin{array}{c}\text { In die } \\
\text { Atmosphäre, } \\
\text { Entnahme durch } \\
\text { (Biomasse 2), }\end{array}$ & $\begin{array}{c}\text { In die } \\
\text { Atmosphäre, } \\
\text { Entnahme } \\
\text { durch (2), } \\
\text { Atmosphäre, } \\
\text { Entnahme durch } \\
\text { (Biomasse 2), }\end{array}$ & ---- \\
\hline $\mathbf{8}$ & Sammlung & $\begin{array}{c}\text { An Punktquellen, } \\
\text { zu (CCU 4) }\end{array}$ & $\begin{array}{c}\text { An } \\
\text { Punktquellen } \\
\text { zu (CCU 4) }\end{array}$ & $\begin{array}{c}\text { An Punktquellen } \\
\text { zu (4) }\end{array}$ & ----- \\
\hline $\mathbf{9}$ & Kreislauf & Nein & Nur (7) & Nur (8) & Ja, über Wasser \\
\hline
\end{tabular}

Der bisher erreichte Erfolg des Umbaus von Energiesystemen ist in seiner zeitlichen Projektion nicht ausreichend um rechtzeitig die Ziele des Vertrages von Paris erreichen zu können. Die nötige konzeptionelle Erweiterung der Entwicklungsplanung erfordert die Umwandlung primärere Elektrizität in transportier- und lagerbare Energieträger auf der Skala des Gesamtinhaltes eines Energiesystems. Ein entstehender technischer Kohlenstoffkreislauf zieht die Ausbildung[23] einer „ $\mathbf{C O}_{\mathbf{2}}$ economy“ nach sich. Damit fällt der Chemie die Aufgabe zu, einen zentralen Teil der Energiesysteme rechtzeitig in einer Qualität so bereit $\mathrm{zu}$ stellen, dass eine kostengünstige und sichere Handhabung stofflicher synthetischer Energieträger auf der Skala der heutigen Petrochemie möglich wird. Die heute noch erheblichen Energieverluste durch ungenügende Optimierung der Reaktionskinetik erheblich zu reduzieren, stellt das vordringliche Forschungsziel dar. Um dies zu erreichen sollte vordringlich die Lücke zwischen konzeptionellem Wissen um katalytische Reaktionen ${ }^{24]}$ und deren technische Realisation geschlossen werden. Die Skalierbarkeit von Rohstoffeinsatz, Materialsynthesen und Prozessen für aus der Grundlagenforschung resultierende wesentliche Verbesserungen ist von höchster Priorität. Die Vielfalt der unmittelbar nötigen chemischen Transformationen (siehe Tabelle 1) erfordert jetzt eine Konzentration auf die damit verbundenen wissenschaftlichen Herausforderungen. Zudem obliegt der Chemie ein breites Betätigungsfeld um die Effizienz der Energiewandlung $\mathrm{zu}$ verbessern. Für neue Lösungen bei Materialien und Prozessen gelten ebenfalls die oben angegebenen Grundbedingungen der Skalierbarkeit. Die Diskussion um technische Effizienzen sollte die nötigen Anstrengungen in chemischer Forschung und Entwicklung für synthetische stoffliche Energieträger nicht behindern. Sie greift zu kurz wenn der Umbau der Energieversorgung in nachhaltige Prozesse auf der Basis erneuerbarer Elektrizität tatsächlich das Ziel der „Energiewende“ sein soll. Das übergeordnete Ziel des Umbaus bleibt eine systemisch effiziente Verwendung von Energie. Dieses Ziel kann nur durch den Nutzer in seinem sozio-ökonomischen Umfeld ${ }^{[22]}$ erreicht werden, die Wissenschaft stellt die dafür nötigen Optionen bereit.

[1] F. Ausfelder, C. Beilmann, M. Bertau, S. Brauninger, A. Heinzel, R. Hoer, W. Koch, F. Mahlendorf, A. Metzelthin, M. Peuckert, L. Plass, K. Rauchle, M. Reuter, G. Schaub, S. Schiebahn, E. Schwab, F. Schuth, D. Stolten, G. Tessmer, K. Wagemann, K. F. Ziegahn, Chemie Ingenieur Technik 2015, 87, 17-89. 
[2] R. Schlögl, Angew. Chem. Int. Ed. 2015, 54, 4436-4439.

[3] aG. Centi, E. A. Quadrelli, S. Perathoner, Energy \& Environmental Science 2013, 6, 1711-1731; bJ. Klankermayer, S. Wesselbaum, K. Beydoun, W. Leitner, Angewandte Chemie-International Edition 2016, 55, 7296-7343.

[4] A. Al-Mamoori, A. Krishnamurthy, A. A. Rownaghi, F. Rezaei, Energy Technology 2017, 5, 834-849.

[5] J. C. Abanades, E. S. Rubin, M. Mazzotti, H. J. Herzog, Energy \& Environmental Science 2017, 10, 2491-2499.

[6] H. Lund, B. V. Mathiesen, Energy 2009, 34, 524-531.

[7] T. Bruhn, H. Naims, B. Olfe-Krautlein, Environmental Science \& Policy 2016, 60, 38-43.

[8] M. Aresta, Coordination Chemistry Reviews 2017, 334, 150-183.

[9] S. Perathoner, S. Gross, E. J. M. Hensen, H. Wessel, H. Chraye, G. Centi, Chemcatchem 2017, 9, 904-909.

[10] B. Smit, Faraday Discussions 2016, 192, 9-25.

[11] E. S. Sanz-Pérez, C. R. Murdock, S. A. Didas, C. W. Jones, Chem. Rev. 2016, 116, 11840-11876.

[12] G. Wilson, M. Trusler, J. Yao, J. S. M. Lee, R. Graham, N. Mac Dowell, R. CuellarFranca, G. Dowson, P. Fennell, P. Styring, J. Gibbins, M. Mazzotti, S. Brandani, C. Muller, R. Hubble, Faraday Discussions 2016, 192, 561-579.

[13] N. Mac Dowell, P. S. Fennell, N. Shah, G. C. Maitland, Nature Climate Change 2017, 7, 243-249.

[14] J. Rockstrom, O. Gaffney, J. Rogelj, M. Meinshausen, N. Nakicenovic, H. J. Schellnhuber, Science 2017, 355, 1269-1271.

[15] C. EEA Indicators, 2017.

[16] A. Azapagic, S. Perdan, Aiche Journal 2014, 60, 3998-4007.

[17] N. M. Haegel, R. Margolis, T. Buonassisi, D. Feldman, A. Froitzheim, R. Garabedian, M. Green, S. Glunz, H. M. Henning, B. Holder, I. Kaizuka, B. Kroposki, K. Matsubara, S. Niki, K. Sakurai, R. A. Schindler, W. Tumas, E. R. Weber, G. Wilson, M. Woodhouse, S. Kurtz, Science 2017, 356, 141-143.

[18] C. Philibert, International Energy Agency, Paris, 2017, p. 65.

[19] Z. W. Seh, J. Kibsgaard, C. F. Dickens, I. B. Chorkendorff, J. K. Norskov, T. F. Jaramillo, Science 2017, 355, 146-+.

[20] A. G. Dana, O. Elishav, A. Bardow, G. E. Shter, G. S. Grader, Angewandte ChemieInternational Edition 2016, 55, 8798-8805.

[21] aY. Ishimoto, A. Kurosawa, M. Sasakura, K. Sakata, International Journal of Hydrogen Energy 2017, 42, 13357-13367; bX. Y. Xu, Y. Liu, F. Zhang, W. Di, Y. L. Zhang, Catalysis Today 2017, 298, 61-68; cM. Reuss, T. Grube, M. Robinius, P. Preuster, P. Wasserscheid, D. Stolten, Applied Energy 2017, 200, 290-302.

[22] B. Truffer, J. Schippl, T. Fleischer, Technological Forecasting and Social Change 2017, 122, 34-48.

[23] E. I. Koytsoumpa, C. Bergins, E. Kakaras, Journal of Supercritical Fluids 2018, 132, 3-16.

[24] aR. Schlögl, Angew. Chem. Int. Ed. 2015, 54, 3465-3520; bG. Ertl, Angewandte Chemie-International Edition 2008, 47, 3524-3535. 